\title{
A deep WSRT 1.4 GHz radio survey of the Spitzer Space Telescope FLSv region $\star$
}

\author{
R. Morganti ${ }^{1}$, M. A. Garrett ${ }^{2}$, S. Chapman ${ }^{3}$, W. Baan ${ }^{1}$, G. Helou ${ }^{3}$, and T. Soifer ${ }^{3}$ \\ 1 Netherlands Foundation for Research in Astronomy, Postbus 2, 7990 AA, Dwingeloo, The Netherlands \\ e-mail: morganti@astron.nl \\ 2 Joint Institute for VLBI in Europe, Postbus 2, 7990 AA, Dwingeloo, The Netherlands \\ 3 California Institute of Technology, MS 320-47, Pasadena, CA 91125, USA
}

Received 13 February 2004 / Accepted 10 May 2004

\begin{abstract}
The First Look Survey (FLS) is the first scientific product to emerge from the Spitzer Space Telescope. A small region of this field (the verification strip) has been imaged very deeply, permitting the detection of cosmologically distant sources. We present Westerbork Synthesis Radio Telescope (WSRT) observations of this region, encompassing a $\sim 1 \mathrm{sq}$. deg field, centred on the verification strip (J2000 RA = 17:17:00.00, Dec $=$ 59:45:00.000). The radio images reach a noise level of $\sim 8.5 \mu \mathrm{Jy}_{\text {beam }}{ }^{-1}-$ the deepest WSRT image made to date. We summarise here the first results from the project, and present the final mosaic image, together with a list of detected sources. The effect of source confusion on the position, size and flux density of the faintest sources in the source catalogue are also addressed. The results of a serendipitous search for H I emission in the field are also presented. Using a subset of the data, we clearly detect H I emission associated with four galaxies in the central region of the FLSv. These are identified with nearby, massive galaxies.
\end{abstract}

Key words. catalogs - galaxies: active - galaxies: starburst - infrared: galaxies - radio continuum: galaxies - surveys

\section{Introduction}

The Spitzer Space Telescope's (formerly SIRTF, the Space Infrared Telescope Facility) First-Look Survey (FLS, http://ssc.spitzer.caltech.edu/fls/), is expected to go two orders of magnitude deeper than any previous, largearea Mid-IR survey. The extragalactic component of the survey is centred on a region that is within the continuous viewing zone of the satellite, in an area of low IR cirrus foreground emission. The survey is composed of two parts a large-area shallow survey covering an area of $2.5^{\circ} \times 2^{\circ}$ and a smaller $\sim 0.75^{\circ} \times 0.3^{\circ}$ strip, referred to as the "verification" survey (or FLSv). The FLSv observations lie within the shallow survey region, and are centred at $\mathrm{RA}=17: 17: 00.00$, Dec $=59: 45: 00.000, \mathrm{~J} 2000$. By employing integration times $\sim 10$ times longer than the shallow survey, the FLSv will reach largely unexplored (mJy and sub-mJy) sensitivity levels in the mid-IR. It is expected that cosmologically distant star forming galaxies will form the dominant population of the faint FLSv sources counts.

The results of the FLS are expected to rapidly enter the public domain, and they are supported by various space and ground-based "follow-up" observations. Parallel observations

* Full Table 1 is only available in electronic form at the CDS via anonymous ftp to cdsarc.u-strasbg.fr $(130.79 .128 .5)$ or via http://cdsweb.u-strasbg.fr/cgi-bin/qcat?]/A+A/424/371 of the FLS in different wave-bands are crucial in order to fully exploit the new and unique infrared data coming from Spitzer. Complimentary optical, radio and sub-mm data have already been (or are in the process of being) collected. Deep radio observations of the field are of particular interest, since it is well known that for local star forming galaxies, there exists a close correlation between their far-IR and radio luminosities (e.g. Helou et al. 1985; Condon 1992). This tight correlation has recently been demonstrated to also apply at cosmological redshifts and mid-IR wavelengths (e.g. Garrett 2002; Elbaz et al. 2002). At sub-mJy levels, various deep radio surveys (e.g. Richards et al. 2000) demonstrate that the radio source counts are dominated by star forming galaxies located at moderate redshifts $(z \sim 0.7)$. There is also significant overlap between the faintest $\mu \mathrm{Jy}$ radio sources and the cosmologically distant $(z \sim 2-4)$ sub-mm (rest frame FIR) population detected in deep, blank field SCUBA observations (e.g. Chapman et al. 2002).

A $1.4 \mathrm{GHz}$ radio survey of the entire FLS region has been made by Condon et al. (2003) using the VLA in the B-array configuration. The final survey image has a resolution of 5 arcsec and reaches an average $(1 \sigma) \mathrm{rms}$ noise level of $23 \mu \mathrm{Jy}$ beam $^{-1}$. A catalogue of 3565 radio components with peak flux densities greater than $115 \mu \mathrm{Jy}$ $(5 \sigma)$ has been compiled and is publicly available at http://www.cv.nrao.edu/sirtf_fls/. 


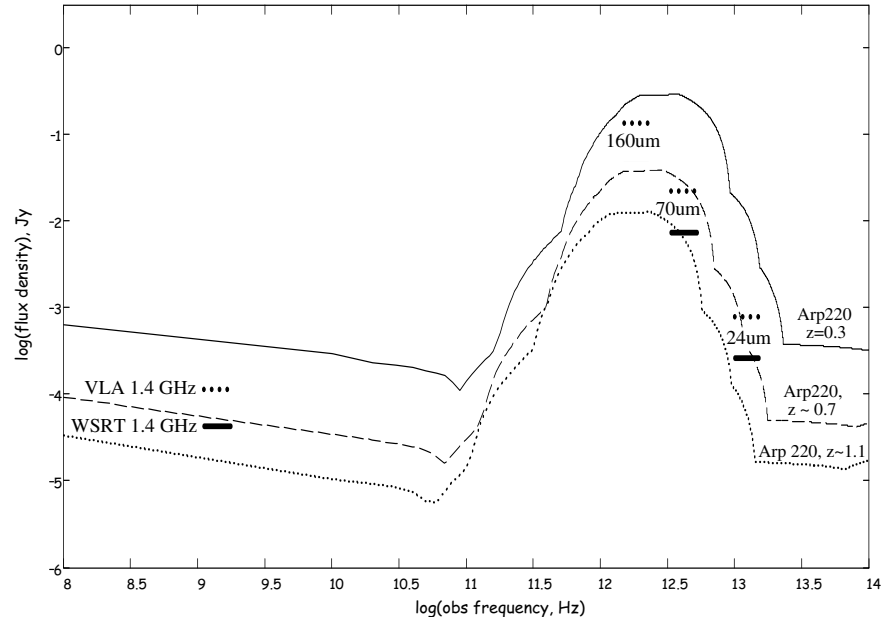

Fig. 1. The SED of Arp 220 (at radio, sub-mm and FIR frequencies) projected to various redshifts $(z=0.3,0.7$ and 1.1). The 5-sigma detection threshold for both the WSRT and VLA 1.4 GHz observations are presented. For comparison, the expected 5-sigma detection threshold levels of Spitzer at 24, 70 and 160 micron for both the shallow survey (dotted line) and deeper verification survey regions (solid lines) are also shown.

Figure 1 shows the observed radio/sub-mm/FIR Spectral Energy Distribution (SED) of the well known Ultra-luminous IR Galaxy, Arp 220, projected to $z \sim 0.3,0.7$, and 1.1 . The 5-sigma sensitivity levels probed by both the VLA and Spitzer FLS and FLSv observations are also presented. The VLA $1.4 \mathrm{GHz}$ survey (Condon et al. 2003) is able to detect Arp 220 type systems out to about $z \sim 0.5$. The shallow Spitzer observations go deeper, capable of detecting Arp 220 out to $z \sim 0.7$. The Spitzer FLSv observations employ much longer integrations times, and are able to go a factor of 3 times deeper (in terms of rms noise level) at 24 and 70 micron (160 micron observations are expected to be confusion limited). The Condon et al. (2003) VLA radio survey, is a relatively poor match to the Spitzer observations, especially within the deeper area of the FLS verification strip.

With a clear need for a much deeper radio survey, over a large area of sky, we started on a project that used the upgraded Westerbork Synthesis Radio Telescope (WSRT) to image a $\sim 1$ sq. deg field, centred on the FLSv strip. The resulting images reach a $(1 \sigma)$ noise level of $\sim 8.5 \mu \mathrm{Jy}$ beam $^{-1}$ - the deepest WSRT image made to date. The 5-sigma detection threshold for the WSRT $1.4 \mathrm{GHz}$ observations presented here are shown in Fig. 1. The WSRT observations can detect Arp 220 like systems out to about $z \sim 0.7$. The FLSv Spitzer observations should be able to detect Arp 220 out to about $z \sim 1$. Only hyper-luminous, star forming systems can be detected beyond $z \sim 1$ by both the Spitzer Space Telescope, VLA and WSRT FLSv survey observations.

Both the VLA and WSRT-FLSv catalogues provide accurate sub-arcsecond, astrometric source positions. These are likely to play a crucial role in the reliable identification of Spitzer sources at other wavelengths. In addition, a comparison of the FIR and radio flux ratios can be used to estimate redshifts for the sources (via the FIR/radio correlation e.g.



Fig. 2. Mosaic pattern of the 7 pointing centres used to image the FLSv. The circles represent the FWHM of the WSRT primary beam. The central field is centred on $\mathrm{RA}(\mathrm{J} 2000)=17: 17: 00.00$ and $\operatorname{Dec}(\mathrm{J} 2000)=59: 45: 00.000)$. The square represents approximately the region used for the extraction of the source catalogue. The region goes from RA $=17: 20: 55$, Dec $=59: 14: 37$ to $\mathrm{RA}=17: 13: 00$, Dec $=$ 60:14:37 (see text for details). The rms noise rises from $\sim 8.5$ in the central area of the image, up to $\sim 20 \mu \mathrm{Jy}_{\text {beam }}^{-1}$ at the edge of the catalogued region.

Carilli \& Yun 2000) or for those sources with measured redshifts, to determine (unobscured) star formation rates and to distinguish between AGN and star formation processes as the main source of the radio and FIR emission.

In this paper, we describe the WSRT $1.4 \mathrm{GHz}$ observations of the FLSv strip, the analysis of the data and the construction of the corresponding (on-line) source catalogue. We also present the first preliminary results of a serendipitous search for $\mathrm{HI}$ emission in the field.

\section{WSRT observations and data reduction}

The total observing time originally requested for the WSRT observations of the FLSv (156 h) was based on the total areal coverage of the elongated FLSv strip. The WSRT observations were made prior to the successful launch of the Spitzer Space Telescope, and since the orientation of the FLSv strip was then unknown, it was decided to image a circular region, centred on the the FLSv. We chose a mosaic of 7 pointings, with a layout similar to that employed by de Vries et al. (2002) in the WSRT observations of the NOAO-N (Bootes) field. The position of the pointing centres for the WSRT FLSv observations are shown in Fig. 2 . The central field is centred on RA(J2000) $=$ 17:17:00.00 and $\operatorname{Dec}(\mathrm{J} 2000)=59: 45: 00.000$. The separation between the grid points was chosen to be $60 \%$ of the $F W H M$ of the primary beam (the WSRT primary beam has a $F W H M \sim$ 35 arcmin at $1.4 \mathrm{GHz}$ ).

The main observations were made during the period May-July 2002, about $16 \mathrm{~h}$ was spent on each pointing. Two additional 12-h observations of the central region, were carried out in Feb. 2003. 


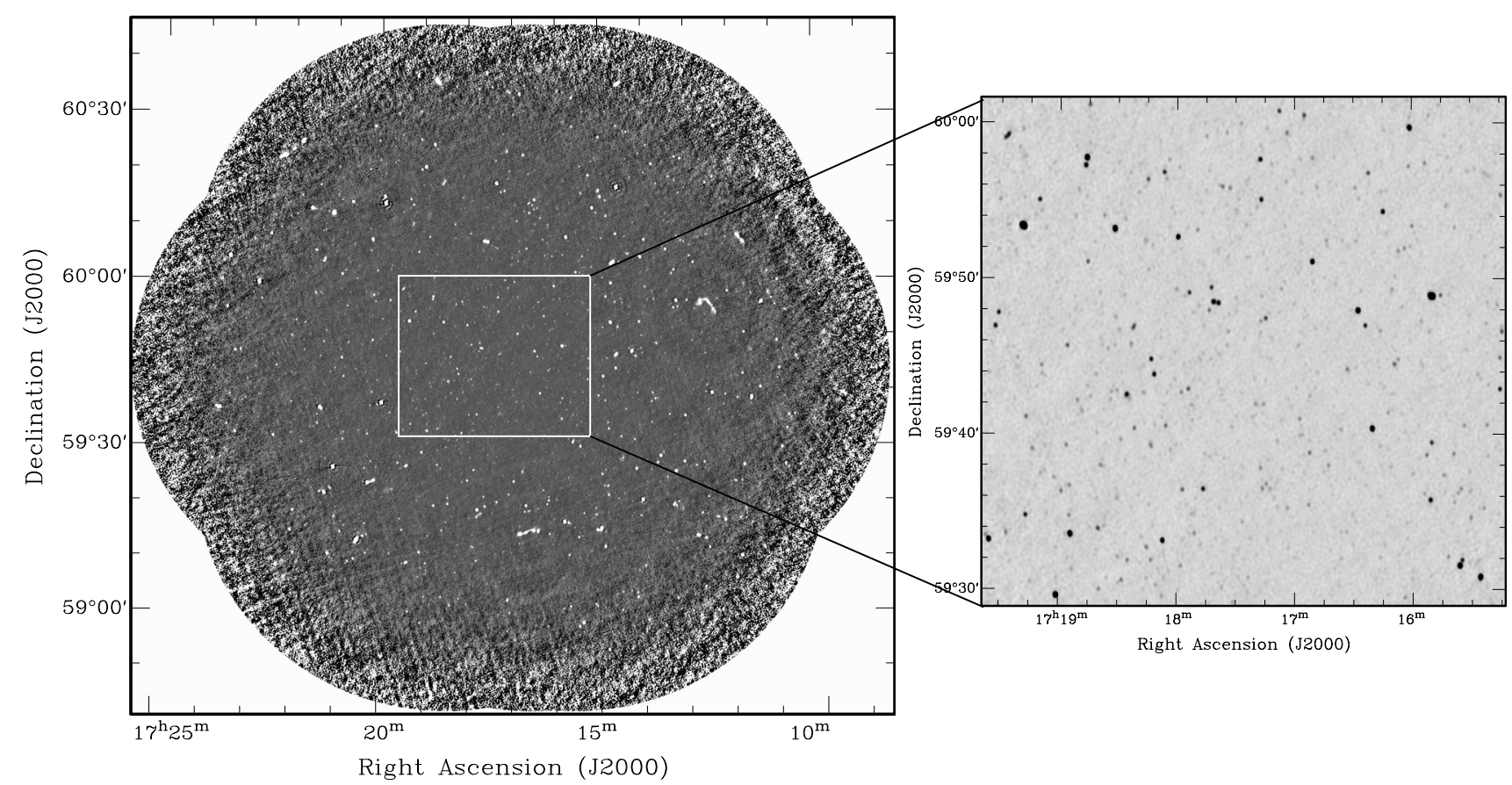

Fig. 3. Left: image of the full WSRT mosaic of the FLSv. Right: the central $30^{\prime} \times 30^{\prime}$ of the mosaic image. The faintest sources visible in this image are at the level of $40 \mu \mathrm{Jy}$ (peak flux density).

The upgraded WSRT is well suited to this type of deep, wide area survey. The new back-end system is capable of imaging out the full field of view of the WSRT antenna primary beam without distortion. The very good sensitivity of the new Multi-Frequency Front End (MFFE) receiver systems, ensures that a single $12 \mathrm{~h}$ observation attains a noise level approaching the expected confusion limit at $1.4 \mathrm{GHz}$.

We made use of the $160 \mathrm{MHz}$ broad-band IF system available at the WSRT. The full band is made up of eight, independently tuned, $20 \mathrm{MHz}$ bands. The 8 bands cover the frequency range between 1420 and $1280 \mathrm{MHz}$. In the last two observations of the central field, however, the 8 bands were centred between 1450 and $1311 \mathrm{MHz}$ - this was found to be the optimum frequency range to use, with respect to RFI considerations. For each of the 8 bands, 128 channels were used (a total of 1024 channels were obtained for the full $160 \mathrm{MHz}$ band) and two polarisation products were recorded. Each $12 \mathrm{~h}$ block was combined with observations (before and after) of an intensity calibrator (typically 3C 147, 3C 48 and/or 3C 286).

The spectral-line mode in which the observations were carried out is the standard mode of operation for continuum observations at the WSRT. This ensures that bandwidth smearing effects are minimised when imaging large fields of view, and also permits the rejection and isolation of narrow RFI spikes, as well as foreground galactic emission. Furthermore, it also permits an interesting by-product, i.e. the possibility of serendipitously detecting HI emission from galaxies in the field (see Sect. 5).

The data were calibrated and reduced using the MIRIAD software package (Sault et al. 1995). Every pointing was mapped using a multi-frequency synthesis approach, where the measurements of all the individual channels are gridded simultaneously in the uv-plane. All the fields were imaged using uniform weighting. Typically three steps of phase selfcalibration were employed, and the CLEAN image deconvolution was performed down to the $3-\sigma$ noise level. In the final deconvolution, frequency dependent effects of the primary beam shape were taken into account (Sault \& Wieringa 1994). All the images were restored with the same synthesised beam of $14^{\prime \prime} \times$ $11^{\prime \prime}\left(\mathrm{PA}=0^{\circ}\right)$. The final maps were corrected for the primary beam response and linearly combined in a mosaic.

\section{Results}

The full mosaic and a more detailed view of the central region are shown in Fig. 3. The final rms noise is $\sim 8.5 \mu \mathrm{Jy}$ beam $^{-1}$ in the central region (increasing to $\sim 20 \mu \mathrm{Jy}_{\text {beam }}{ }^{-1}$ at $\sim 30 \mathrm{arcmin}$ from the centre). This is the deepest image produced with the WSRT at $1.4 \mathrm{GHz}$.

The automatic extraction of the sources was done using the algorithm SFIND 2.0 available inside MIRIAD. A full description of the algorithm as applied to radio images, as well as a comparison with other algorithms, is given by Hopkins et al. (2002). This routine incorporates a statistically robust method for detecting sources, called the "False Discovery Rate". The details of this method are given in Benjamini \& Hochberg (1995).

Here we only briefly summarise the SFIND 2.0 method. The algorithm estimates the noise across the image. In our case the noise is mainly a function of the radial distance from the centre. The values of the local noise for each detected source are given in the last column of Table 1. Each pixel is assigned a probability that it is drawn from the background noise. A cutoff threshold on this probability is then estimated, based on the percentage (chosen here to be $0.1 \%$ ) of false detections of 
Table 1. Example of the radio catalogue. The complete table of 1048 sources can be found in electronic format at CDS in the WSRT-FLSv page available at http://www.astron.nl/wsrt/WSRTsurveys/WFLS. The individual columns are as follows: right ascension (hours, minutes, seconds), Declination (degree, arcminutes and arcseconds), integrated flux density (mJy), major and minor axes (in arcsecond) and position angle (in degrees), local background rms (mJy, see text for details). Major and minor axes and position angle of the sources are not deconvolved for the synthesised beam.

\begin{tabular}{lcccccc}
\hline \hline $\begin{array}{l}\text { RA } \\
(\mathrm{J} 2000)\end{array}$ & $\begin{array}{c}\text { Dec } \\
(\mathrm{J} 2000)\end{array}$ & $\begin{array}{c}\text { Flux density } \\
\mathrm{mJy}\end{array}$ & $\begin{array}{c}\Theta_{\text {maj }} \\
\operatorname{arcsec}\end{array}$ & $\begin{array}{c}\Theta_{\min } \\
\operatorname{arcsec}\end{array}$ & $\begin{array}{c}\text { PA } \\
\text { degrees }\end{array}$ & $\begin{array}{c}\mathrm{rms}_{\text {backg }} \\
\mathrm{mJy}\end{array}$ \\
\hline 17:20:55.927 & $59: 26: 15.26$ & 0.267 & 12.8 & 11.2 & -4.8 & 0.0155 \\
17:20:55.311 & $59: 26: 45.79$ & 0.260 & 17.9 & 12.9 & -74.8 & 0.0195 \\
17:20:55.850 & $59: 35: 41.65$ & 0.171 & 16.7 & 12.3 & -40.8 & 0.0189 \\
17:20:57.189 & $59: 54: 45.97$ & 0.101 & 15.4 & 10.3 & 25.3 & 0.0149 \\
17:20:56.790 & $59: 56: 46.29$ & 0.116 & 19.6 & 12.3 & -9.6 & 0.0132 \\
17:20:50.551 & $59: 15: 12.81$ & 1.630 & 14.7 & 11.2 & 10.5 & 0.0273 \\
17:20:55.200 & $59: 49: 50.67$ & 0.131 & 16.4 & 11.0 & -24.1 & 0.0157 \\
17:20:53.402 & $59: 41: 16.88$ & 0.130 & 29.8 & 11.5 & 2.5 & 0.0132 \\
17:20:53.421 & $59: 44: 18.04$ & 0.091 & 15.5 & 11.4 & -24.9 & 0.0149 \\
17:20:50.753 & $59: 32: 55.72$ & 0.233 & 17.4 & 13.5 & 30.9 & 0.0163 \\
17:20:52.516 & $59: 48: 10.41$ & 0.224 & 15.7 & 13.1 & -0.4 & 0.0139 \\
\hline
\end{tabular}

source pixels. The sources are then selected on this basis and their position, sizes, peak and total flux density are measured. To produce the catalogue presented here, we have used the option of selecting sources for which the peak-pixel is above the estimated threshold.

The extraction of the sources was done in the inner $1 \mathrm{sq}$. deg area (see Fig. 2). In this area 1048 sources were detected. The estimated background noise reaches $\sim 8 \mu \mathrm{Jy}_{\text {beam }}{ }^{-1}$ or better in the central region, comparable to the expected theoretical noise $\left(\sim 6.5 \mu \mathrm{Jy} \mathrm{beam}^{-1}\right.$ for uniformly weighted data). The variation in the background noise level shows the expected trend, rising with increasing distance from the centre. At $30 \mathrm{arcmin}$ from the centre of the field, the measured noise level increases to $\sim 20 \mu \mathrm{Jy}_{\text {beam }}{ }^{-1}$. An analysis of the ratio between the peak flux density of the source and the background noise estimated around that source, shows that we are reaching what would correspond to a $\sim 5 \sigma$ detection level in our catalogue. The number of detected sources compares well with that expected from the known source counts (see e.g. Richards et al. 1998). A simple estimate, considering the characteristics of the noise in our image (increasing from the centre to the outer regions), indicates that we should expect about 1150 sources at the $5 \sigma$ level. The remaining discrepancy can be due to the fact that some of the sources will be resolved by VLA observations into multiple components.

For the detected sources, SFIND 2.0 provides as output a number of parameters. They are listed in Table 1, where part of the catalogue is presented to illustrate the format. The full version of the catalogue, as well as the complete mosaic (in FITS format), can be down loaded through the WSRT-FLSv page at http://www . astron.nl/wsrt/WSRTsurveys/WFLS and in electronic form at CDS.

We note in passing, that the more "classical" algorithm for source extraction, IMSAD, was also tried on the FLSv field. This algorithm (also available in MIRIAD, see Prandoni et al. 2000 for details) selects all the connected regions of pixels above a given flux density threshold (taken to be 5- $\sigma$ for the mosaic field of the FLSv). These regions are taken as the sources present in the image above a certain flux limit. The algorithm then performs a two-dimensional Gaussian fit of the flux distribution for each source. Although the catalogues produced with the two different methods widely overlap, IMSAD proved to be less robust, compared to SFIND 2.0, against false detections and also more prone to fail to detect group of sources when they are separated by about a beam size.

Finally, there are a few sources that are clearly resolved with the $\sim 14^{\prime \prime}$ WSRT beam. Some examples of well resolved sources are presented in Fig. 4.

\section{Confusion}

The position of the faintest sources in the catalogue may be affected by the effects of source confusion. The usual rule of thumb is that confusion becomes a problem when the source counts reach $1 / 30$ per resolving beam. At this level the positions, fluxes and sizes of the faintest sources deviate from their true position since the measurement is also affected by the random distribution of still fainter, unresolved sources that are not detected individually. This sea of unresolved background sources can therefore modify the measurements for the faintest sources in our catalogue. Hogg (2001) simulated images of crowded fields, assuming various slopes for the differential source counts. Following Hogg's definition, the source counts in our survey reach about $1 / 44$ per resolving beam. Taking a slope of -1.5 (somewhat steeper than the measured slope e.g. Richards 2000), we estimate from Hogg's Fig. 4, that due to the effects of source confusion, the median error in the measured positions of our faintest sources will typically be $\sim 0.2$ of the FWHM of WSRT resolving beam. Occasionally (about $10 \%$ of the time) sources will have larger errors, about 0.4 of the $F W H M$. Thus for the faintest sources in our catalogue, positions of some sources could, in principle, be in error by as 



Fig. 4. Examples of clearly resolved sources in the WSRT mosaic. Contour levels are $30,60,120, \ldots . \mu \mathrm{Jy} \mathrm{beam}^{-1}$. With the exception of the last three, all sources appear to be extended also in the VLA catalogue of Condon et al. (2003, see also their Figs. 5 to 13).

much as 6 arcsec. In a similar way, confusion can also affect the measured source sizes. Flux densities errors can also arise, the median error is $\sim 5 \%$ but occasionally these can be as much as $30 \%$.

It should be noted that the errors induced by source confusion are confined to the deepest, central areas of the WSRT image. At 15 arcmin from the central field, the noise rises to $11 \mu \mathrm{Jy} \mathrm{beam}^{-1}$, the source counts fall to $\sim 1 / 63$ per resolving beam, and the effects of confusion are very much reduced. At about 22 arcmin from the central field the noise rises to $15 \mu \mathrm{Jy}$ beam $^{-1}$, the source counts fall to $\sim 1 / 100$ and the effects of confusion become negligible.
The WSRT FLS FITS file is in the public domain. Astronomers using this image to cross-identify sources at the 3 sigma level, should be aware that the effects of confusion on estimated positions, fluxes etc. will be much larger (the source counts rise to $\sim 1 / 22$ per resolving beam in the central part of the field), making detailed comparisons unreliable.

\section{Positional accuracy}

As we have discussed in the previous section, the positional errors associated with the faintest sources in our catalogue are likely dominated by the effects of source confusion. However, 
for the brighter sources it is still important to compare the positions of our extracted sources with those from the VLA catalogue of Condon et al. (2003). Given the difference (more than a factor 2) in resolution, this comparison is not completely straightforward. In particular, the number of resolved sources is much higher for the VLA observations, and therefore the offset between the two may reflect the different morphologies of the sources at the two resolutions.

In Fig. 5 we show the distribution of the offset in RA and Dec as obtained from the large (1-degree) region. The size of the symbols is proportional to the peak flux. Similar results are found when comparing the sources in the inner (25-arcmin) region. The median offset is about 1 arcsec. As expected, weaker sources tend to have a larger offset. Following the relation discussed in Prandoni et al. (2000) and Condon (1998), the formal positional accuracy expected in the case of our observations is $\sim 1.5^{\prime \prime}$ for a point source at the limit of the survey $(5 \sigma)$.

However, as shown from the cross plotted in Fig. 5, a systematic offset of about 0.5 arcsec has been found in declination. In RA the offset is less than $0.1^{\prime \prime}$. This offset in declination corresponds to less than a $1 / 20$ of the synthesised beam width. Nevertheless, given that this offset seems to be independent of the flux density of the sources, it probably reflects the typical astrometric accuracy the WSRT can achieve, using standard calibration techniques.

No correction for the offset has been applied to the coordinates presented in the catalogue.

\section{A serendipitous search for $\mathrm{HI}$ emission}

As described in Sect. 2, the data presented here included observing bands that were set to frequencies below $1421 \mathrm{MHz}$, the rest-frequency of neutral hydrogen emission. Indeed, the standard continuum WSRT set-up is well suited for a serendipitous search for $\mathrm{H} \mathrm{I}$ emission (and also H I absorption, if strong sources are present in the field). The frequency range of our observations, together with the large number of channels employed for each $20 \mathrm{MHz}$ band, thus permitted us to search for serendipitous H I emission from galaxies in the FLSv field.

We selected a subset of the observations, specifically the $2 \times 12 \mathrm{~h}$ centred on the FLSv, to look for possible serendipitous $\mathrm{HI}$ emission. After subtracting the continuum CLEAN components from the (self-calibrated) visibility data sets, the data were Hanning smoothed to suppress Gibbs effects that are inherent to digital correlators.

A region covering about $40^{\prime \prime}$ on a side was imaged using a robust weighting equal to 1 . The synthesised beam is $24^{\prime \prime} \times$ $20^{\prime \prime}$ (in $\mathrm{PA}=1.4^{\circ}$ ). Of this region, a spectral-line data cube of 410 channels with a velocity resolution of $60 \mathrm{~km} \mathrm{~s}^{-1}$ width was generated. It should be noted that a resolution of $60 \mathrm{~km} \mathrm{~s}^{-1}$ is good enough to distinguish between single or multiple galaxies, and even measure the galaxies rotation velocity for massive H I systems. A second data set, with a velocity resolution further smoothed to $100 \mathrm{~km} \mathrm{~s}^{-1}$ was generated for comparison purposes. The entire data cube for both data sets covered the velocity range from 0 to $24600 \mathrm{~km} \mathrm{~s}^{-1}$. The typical $(1 \sigma)$ noise level obtained in each $60 \mathrm{~km} \mathrm{~s}^{-1}$ channel was about $0.12 \mathrm{mJy} /$ beam.



Fig. 5. Distribution of the position offsets (in right ascension and declination) between the sources in common to the WSRT and the VLA in the 1-degree region of the WSRT mosaic. The size of the symbols is proportional to the flux of the source.

The final data cube was searched for $\mathrm{HI}$ emission using both a visual inspection of the data and an automatic detection routine (kindly provided by $\mathrm{T}$. Oosterloo). The candidate detections were then further inspected for optical counterparts using deep optical images obtained with the NOAO telescope ( $5 \sigma$ depth limiting magnitude of $R=25.5$ (Vega), Fadda et al. 2004) and publicly available at http://ssc.spitzer.caltech.edu/fls/extragal/ noaor.html. Four H I detections were found in both the 60 and $100 \mathrm{~km} \mathrm{~s}^{-1}$ data cubes. Figure 6 shows these results, with the total H I intensity of the confirmed detections superimposed upon the optical image. The parameters of the HI detections are summarised in Table 2. All four H I detections are identified with nearby, massive galaxies. A similar number of serendipitous HI detections were also found (using the same technique) in a deep survey (of similar extent) performed within the NOAO-N "Bootes" field (Morganti \& Garrett 2002). The results of a search for serendipitous HI detections from the full mosaic data set, will be presented in a forthcoming paper. No HI absorption was detected towards any of the sources in the 40" FLSv field.

Finally, an estimate using the HI mass function (Zwaan 2000) suggests that every field observed with a similar depth should contain about 5 objects with detectable HI emission. The majority of the expected objects will be $M_{\star}$ galaxies, i.e. galaxies with an amount of neutral hydrogen between $10^{9}$ to $10^{10} M_{\odot}$. The bias toward the detection of massive systems, is partly due to the relatively coarse velocity resolution that is obtained using the "default" continuum set-up. 
Table 2. H I detections. The H I masses are estimated assuming $H_{0}=70 \mathrm{~km} \mathrm{~s}^{-1} \mathrm{Mpc}^{-1}$.

\begin{tabular}{lccccc}
\hline \hline Name & RA & Dec & Vel & H I Mass \\
& J2000 & J2000 & $\mathrm{km} \mathrm{s}^{-1}$ & $M_{\odot}$ & $\begin{array}{c}\mathrm{S}_{1.4 \mathrm{GHz}} \\
\mu \mathrm{Jy}\end{array}$ \\
\hline $\mathrm{J} 171441+595052$ & $17: 14: 41$ & $59: 50: 52$ & 5474 & $2.1 \times 10^{9}$ & 87 \\
$\mathrm{~J} 171603+592333$ & $17: 16: 03$ & $59: 23: 33$ & 7881 & $2.6 \times 10^{9}$ & - \\
$\mathrm{J} 171612+594026$ & $17: 16: 12$ & $59: 40: 26$ & 16921 & $6.1 \times 10^{9}$ & 43 \\
$\mathrm{~J} 171729+594757$ & $17: 17: 29$ & $59: 47: 57$ & 23551 & $1.3 \times 10^{9}$ & - \\
\hline
\end{tabular}

$\mathrm{J} 171441+595052$

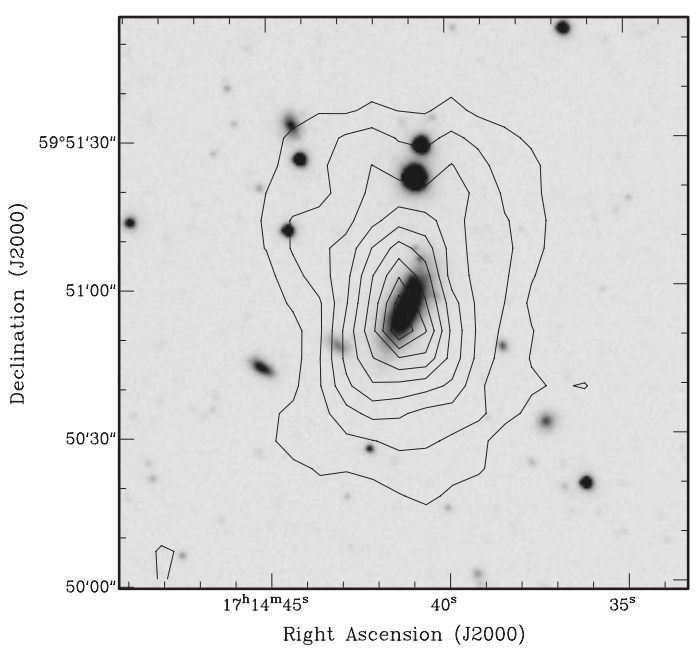

$\mathrm{J} 171612+594026$

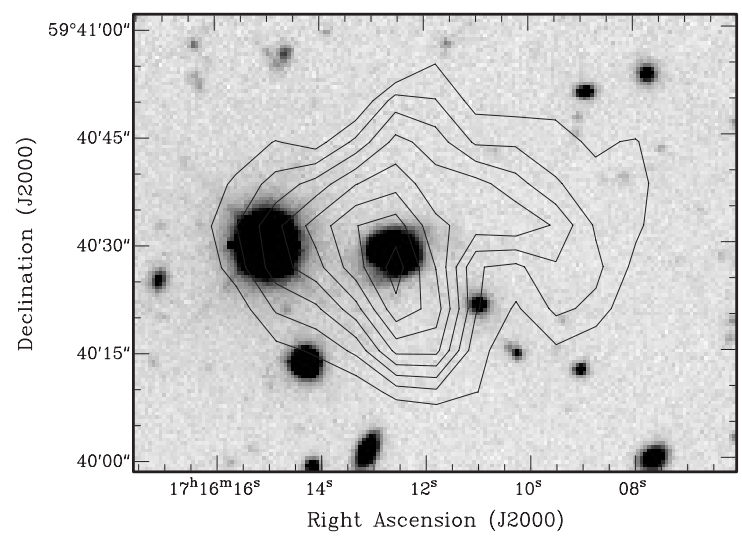

J171603+592333

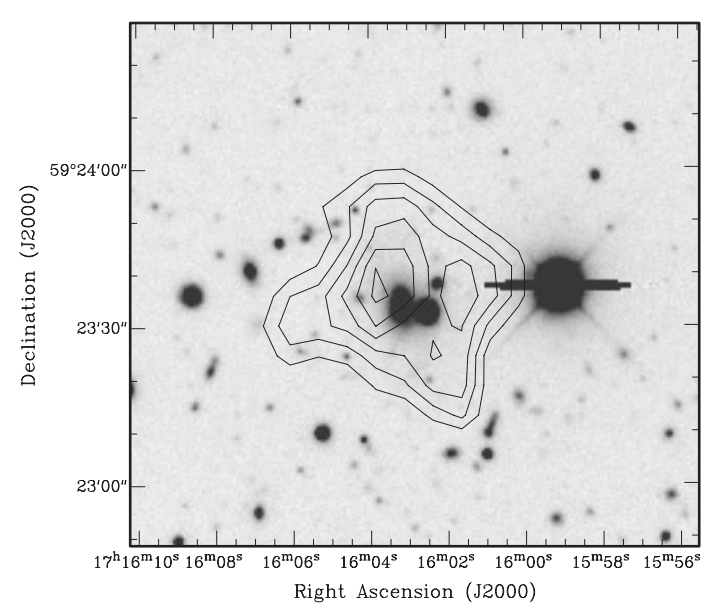

J171729+594757

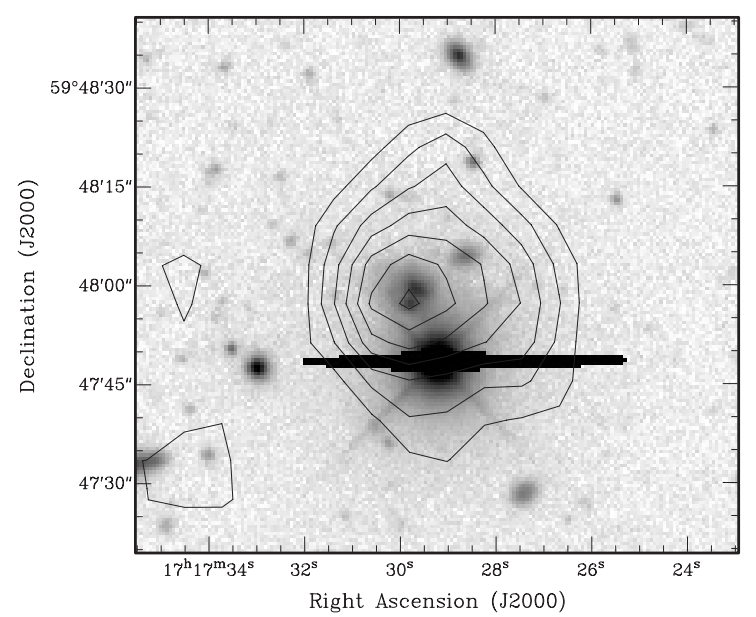

Fig. 6. H I total intensity contours superimposed on optical images of the four H I detections in the central region of the FLSv field. The contour levels are: for J171441+595052 $2.2 \times 10^{20}$ to $2.9 \times 10^{21} \mathrm{~cm}^{-2}$ in steps of $3.2 \times 10^{20} \mathrm{~cm}^{-2}$; for J171603+592333 $4.0 \times 10^{20}$ to $1.6 \times 10^{21} \mathrm{~cm}^{-2}$ in steps of $2.2 \times 10^{20} \mathrm{~cm}^{-2}$; for J171612+594026 $1.8 \times 10^{20}$ to $7.0 \times 10^{20} \mathrm{~cm}^{-2}$ in steps of $7.3 \times 10^{19} \mathrm{~cm}^{-2}$; for J171729+594757 $1.8 \times 10^{20}$ to $1.4 \times 10^{21} \mathrm{~cm}^{-2}$ in steps of $1.7 \times 10^{20} \mathrm{~cm}^{-2}$.

\section{Follow up observations}

Compared to other deep field radio observations (e.g. the much smaller regions covered by the Hubble Deep Field surveys), the VLA and WSRT-FLSv catalogues cover significant areas of sky with excellent microJy sensitivity. In particular, the deep radio observations presented here, will ensure significant overlap between the radio and FIR Spitzer FLSv observations out to at least $z \sim 1$. Hyper-luminous star forming galaxies will be detected at even higher redshifts.

Spectroscopic and photometric redshift campaigns in the region of the FLSv are already underway. Successful SCUBA observations have been carried out using a radio selection combined with optical red colours (Frayer et al. 2004). 
The complementarity between the Spitzer and WSRT/VLA observations will enable the first detailed study of the FIR-Radio correlation to be made, out to at least $z \sim 1.5$. While there is already good evidence that the correlation persists out to at least $z \sim 1$ (Garrett 2002), the Spitzer and WSRT observations may uncover more subtle deviations or possible large scale trends in the relation, as a function of redshift or galaxy (starburst) type. In addition, sources that deviate from the relation will be easily identified as rare and interesting AGN, in a radio and FIR field that is otherwise dominated by star forming systems.

These wide area VLA and WSRT radio surveys will also be important in acting as a finding chart for very deep, high resolution, wide-field VLBI observations of the FLSv region. By using the technique of full-beam calibration (Garrett et al. 2003), it should be possible to image several dozen faint (radio-loud) AGN in the field with full microJy sensitivity and milliarcsecond resolution. For the first time, it will be possible to conduct a large-scale census of the milliarcsecond structures of these submJy and microJy radio sources. Astrometric positions of the sources detected will be measured with reference to the IERS reference frame with a precision of a milliarcsecond or better. The first results are already presented in Wrobel et al. (2004). The VLBI observations will also provide an independent and unambiguous check on the AGN content of the field.

Finally, we note in passing that even deeper serendipitous H I observations are possible with the WSRT's standard $L$-band continuum set-up. In particular, the $21-\mathrm{cm}$ band can reach $\sim 1160 \mathrm{MHz}$ (albeit with a less favourable RFI environment). By selecting frequencies at the edge of the band, one can, in principle, explore serendipitous H I emission up to redshift of $z=0.22$.

Acknowledgements. We thank T. Oosterloo for his help with the $\mathrm{HI}$ data and in particular for providing the routine for the automatic detection of HI candidates. The authors also thank the referee for many useful comments that have helped to improve the content of the paper. The WSRT is operated by the ASTRON
(Netherlands Foundation for Research in Astronomy) with support from the Netherlands Foundation for Scientific Research (NWO).

\section{References}

Benjamini, Y., \& Hochberg, Y. 1995, J. R. Stat. Soc. B, 57, 289

Condon, J. J. 1992, ARA\&A, 30, 575

Condon, J. J. 1998, in New horizons from multi-wavelength sky surveys, ed. B. J. McLean, D. A. Golombek, J. J. E. Hayes, \& H. E. Payne, Proc. IAU Symp., 179, 19

Condon, J. J., Cotton W. D., Yin Q. F., et al. 2003, AJ, 125, 2411

Carilli, C. L., \& Yun, S. M. 2000, ApJ, 530, 618

Chapman, S. C., Richards, E. A., Lewis, G. F., Wilson, G., \& Barger, A. J. 2001, ApJ, 548, L147

Chapman, S. C., Lewis, G. F., Scott, D., Borys, C., \& Richards, E. 2002, ApJ, 570, 637

de Vries, W. H., Morganti, R., Röttgering, H. J. A., et al. 2002, AJ, 123,1784

Elbaz, D., Cesarsky, C. J., Chanial, P., et al. 2002, A\&A, 384, 848

Fadda, D., Jannuzi, B., Ford, A., \& Storrie-Lombardi, L. J. 2004, AJ, in press [arXiv: astro-ph/0403490]

Frayer, D. T., et al. 2004, ApJS Spitzer Special Edition, in press [arXiv: astro-ph/0406351]

Garrett, M. A. 2002, A\&A, 384, L19

Garrett, M. A., Wrobel, J. M., \& Morganti, R. 2003, NewAR, 47, 385 [arXiv: astro-ph/0301465]

Helou, G., Soifer, B. T., \& Rowan-Robinson, M. 1985, ApJ, 298, L7

Hogg, D. W. 2001, ApJ, 112, 1207

Hopkins, A. M., Miller, C. J., Connolly, A. J., et al. 2002, AJ, 123, 1086

Morganti, R., \& Garrett, M. A. 2002, ASTRON Newslett., 17, 6 www.astron.nl/astron/newsletters/2002_1

Prandoni, I., Gregorini, L., Parma, P., et al. 2000, A\&AS, 146, 41

Richards, E. A., Kellermann, K. I., Fomalont, E. B., Windhorst, R. A., \& Partridge, R. B. 1998, AJ, 116, 1039

Richards, E. A. 2000, ApJ, 533, 611

Sault, R. J., \& Wieringa, M. H. 1994, A\&AS, 108, 585

Zwaan, M. 2000, Ph.D. Thesis, University of Groningen

Wrobel, J. M., Garrett, M. A., Condon, J. J., \& Morganti, R. 2004, AJ, in press [arXiv:astro-ph/0404007] 